\title{
Performance Issues for Vertex Elimination Methods in Computing Jacobians using Automatic Differentiation
}

\author{
Mohamed Tadjouddine ${ }^{1}$, Shaun A. Forth ${ }^{1}$, John D. Pryce ${ }^{2}$, and John K. Reid ${ }^{3}$ \\ 1 Applied Mathematics \& Operational Research, ESD \\ Cranfield University (RMCS Shrivenham), Swindon SN6 8LA, UK \\ \{M.Tadjouddine, S.A.Forth\}@rmcs.cranfield.ac.uk \\ 2 Computer Information Systems Engineering, DOIS \\ Cranfield University (RMCS Shrivenham), Swindon SN6 8LA, UK \\ J.D.Pryce@rmcs.cranfield.ac.uk \\ 3 JKR Associates, 24 Oxford Road, Benson, Oxon OX10 6LX, UK \\ jkr@rl.ac.uk
}

\begin{abstract}
In this paper, we present first results from EliAD, a new automatic differentiation tool. EliAD uses the Elimination approach for Automatic Differentation first advocated by Griewank and Reese [Automatic Differentiation of Algorithms, SIAM (1991), 126-135]. EliAD implements this technique via source-transformation, writing new Fortran code for the Jacobians of functions defined by existing Fortran code. Our results are from applying EliAD to the Roe flux routine commonly used in computational fluid dynamics. We show that we can produce code that calculates the associated flux Jacobian approaching or in excess of twice the speed of current state-of-the-art automatic differentiation tools. However, in order to do so we must take into account the architecture on which we are running our code. In particular, on processors that do not support out-of-order execution, we must reorder our derivative code so that values may be reused while in arithmetic registers in order that the floating point arithmetic pipeline may be kept full.
\end{abstract}

\section{Introduction}

In scientific computation, there is a frequent need to compute first derivatives of a function represented by a computer program. One way to achieve this is to use Automatic Differentiation (AD) [1-3], which allows for the computation of derivatives of a function represented by a computer program. The most efficient way to implement $\mathrm{AD}$ in terms of run-time speed is usually source transformation; here the original code is augmented by statements that calculate the needed derivatives. ADIFOR [4], Odyssée [5], and TAMC [6] are well-established tools for this which make use of the standard forward and reverse modes of AD.

We have developed a new AD tool EliAD [7,8] which also uses source transformation. EliAD is written in Java and uses a parsing front-end generated by the ANTLR tool [9]. In contrast to the AD tools listed above, EliAD uses the 
vertex elimination approach of Griewank and Reese [2,10], later generalised to edge and face eliminations by Naumann $[11,12]$. Here, we consider only the vertex elimination approach, which typically needs less floating point operations to calculate a Jacobian than the traditional forward and reverse methods implemented by ADIFOR, Odyssée or TAMC. We introduce the vertex elimination approach in Sect. 2.

In Sect. 3 we present results of applying our tool to the Roe flux [13] computation which is a central part of many computational fluid dynamics codes. We used various ways to sequence the elimination. We ran the resulting derivative codes on various machine/compiler combinations and at various levels of compiler optimisation. We found that execution times were not always in proportion to the number of floating point operations and that this effect was very machine-dependent.

Section 4 discusses the performance of certain elimination strategies regarding such machine dependent issues as cache and register utilisation and then points out the importance of statement ordering on certain processors.

\section{Automatic Differentiation by Elimination Techniques}

$\mathrm{AD}$ relies on the use of the chain rule of calculus applied to elementary operations in an automated fashion. The input variables $x$ with respect to which we need to compute derivatives are called independent variables. The output variables $y$ whose derivatives are desired are called dependent variables. A variable which depends on an independent variable, and on which a dependent variable depends, is called an intermediate variable.

To illustrate the elimination approach, consider the code fragment comprising four scalar assignments in the left of

$$
\begin{array}{ll}
x_{3}=\phi_{3}\left(x_{1}, x_{2}\right) & \nabla x_{3}=c_{3,1} \nabla x_{1}+c_{3,2} \nabla x_{2} \\
x_{4}=\phi_{4}\left(x_{2}, x_{3}\right) & \nabla x_{4}=c_{4,2} \nabla x_{2}+c_{4,3} \nabla x_{3} \\
x_{5}=\phi_{5}\left(x_{1}, x_{3}\right) & \nabla x_{5}=c_{5,1} \nabla x_{1}+c_{5,3} \nabla x_{3} \\
y=\phi_{6}\left(x_{4}, x_{5}\right) & \nabla y=c_{6,4} \nabla x_{4}+c_{6,5} \nabla x_{5} .
\end{array}
$$

Denoting

$$
\nabla x_{i}=\left(\frac{\partial x_{i}}{\partial x_{1}}, \frac{\partial x_{i}}{\partial x_{2}}\right) \text { and } c_{i, j}=\frac{\partial \phi_{i}}{\partial x_{j}},
$$

we may use standard rules of calculus to write the linearised equations to the right of (1). These linearised equations describe the forward mode of $\mathrm{AD}$ and equivalent code would be produced by ADIFOR or TAMC enabling Jacobian calculation on setting $\nabla x_{1}=(1,0)$ and $\nabla x_{2}=(0,1)$. The Computational Graph (CG) for derivative calculation is sketched in Fig. 1. Vertices 1, 2 represent the independents $x_{1}, x_{2}$; vertices $3,4,5$ the intermediate variables $x_{3}, x_{4}, x_{5}$; and vertex 6 the dependent $y$. The edges are labelled with the local derivatives of the equivalent code statements given to the right of (1) as shown. The corresponding 


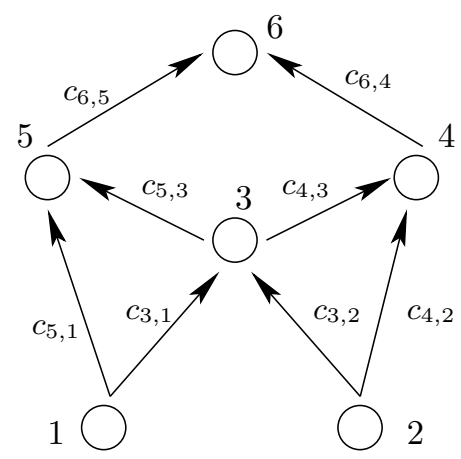

Fig. 1. An example computational graph

matrix representation gives a linear system, to solve for the derivatives, with zero entries in the matrix omitted for clarity:

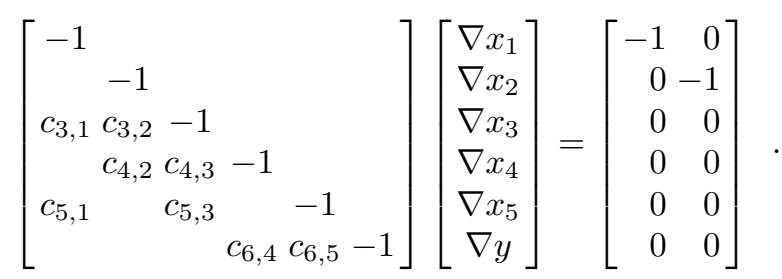

We may interpret the elimination approach via either the computational graph or the matrix representation. Via the graph the Jacobian $\frac{\partial y}{\partial\left(x_{1}, x_{2}\right)}$ is determined by eliminating intermediate vertices from the graph until it becomes bipartite, see $[2,10,11]$. For example vertex 5 may be eliminated by creating new edges $c_{6,1}=c_{6,5} \times c_{5,1}, c_{6,3}=c_{6,5} \times c_{5,3}$ and then deleting vertex 5 and all the adjacent edges. We might then eliminate vertex 4 then 3 , termed a reverse ordering.

In terms of the matrix representation, vertex elimination is equivalent to choosing a diagonal pivot from rows 3 to 5 , in some order. At each step we eliminate all the coefficients under that pivot. For example by choosing row 5 as the pivot row we add the multiple $c_{6,5}$ of row 5 to row 6 and so produce entries $c_{6,1}=c_{6,5} \times c_{5,1}, c_{6,3}=c_{6,5} \times c_{5,3}$ in row 6 . We see that this is identical to the above computational graph description. By then choosing rows 4 and 3 as pivots we are left with the Jacobian in elements $c_{61}$ and $c_{62}$.

There are as many vertex elimination sequences as there are permutations of the intermediate vertices. We choose a sequence using heuristics from sparse matrix technology aimed at reducing fill-in, such as the Markowitz criterion studied in $[10,11]$. By reducing fill-in such techniques have the desired side-effect of choosing an ordering which minimises the number of floating point operations at each elimination step. 


\section{$3 \quad$ Numerical Results}

A typical application is the Roe flux routine [13]. This computes the numerical fluxes of mass, energy and momentum across a cell face in a finite-volume compressible flow calculation. Roe's flux takes as input 2 vectors of length 5 describing the flow either side of a cell face and returns as output a length 5 vector for the numerical flux (139 lines of code). We seek the $5 \times 10$ Jacobian matrix.

We used EliAD to differentiate the Roe flux code with different vertex elimination orderings. Those orderings are Forward, Reverse, Markowitz (Mark), VLR, Markowitz Reverse Bias (Mark RB), and VLR Reverse Bias (VLR RB) eliminations; see for instance $[11,14]$ for details. The reverse-bias variants resolve ties when there are several nodes of the same cost by taking the one that appears last in the original statement order [14].

From the input code we generated derivative codes in one of the two following manners.

1. Statement level: local derivatives are computed for each statement, no matter how many variables $x_{i}$ appear in its right-hand side.

2. Code list: local derivatives are computed for each statement in a rewritten code that has at most two variables $x_{i}$ in each right-hand side.

Then, we applied the different vertex elimination strategies to the resulting computational graphs (62 intermediate vertices for the first case and 208 intermediates for the second case). We ran the subsequent derivative codes on the following platforms: Silicon Graphics (SGI), Compaq Alpha (ALP), and two SUN machines with different compilers denoted by SUN, NAG and FUJ. The various processor/compiler combinations are described in Table 1.

Table 1. Platforms (processors and compilers)

\begin{tabular}{|c|c|c|c|c|c|c|}
\hline Label & Processor & $\mathrm{CPU}$ & L1-Cache & L2-Cache & Compiler & Options \\
\hline SGI & R12000 & $300 \mathrm{MHz}$ & $64 \mathrm{~KB}$ & $8 \mathrm{MB}$ & f90 MIPSPro 7.3 & -Ofast \\
\hline ALP & EV6 & $667 \mathrm{MHz}$ & $128 \mathrm{~KB}$ & $8 \mathrm{MB}$ & Compaq f95 5.4 & $-\mathrm{O} 5$ \\
\hline SUN & Ultra10 & $440 \mathrm{MHz}$ & $32 \mathrm{~KB}$ & $2 \mathrm{MB}$ & Workshop f90 6.0 & -fast \\
\hline NAG & Ultra10 & $440 \mathrm{MHz}$ & $32 \mathrm{~KB}$ & $2 \mathrm{MB}$ & Nagware f95 4.0a & -O3 -native \\
\hline FUJ & Ultra1 & $143 \mathrm{MHz}$ & $32 \mathrm{~KB}$ & $0.5 \mathrm{MB}$ & Fujitsu f90 5.0 & -Kfast \\
\hline
\end{tabular}

Table 2 shows the CPU times required by the calculation of the original function on the different platforms.

Table 3 summarises the ratio between the timings of the Jacobian and the original function by using the different methods and platforms. Methods starting with $\mathrm{VE}$ are those using a vertex elimination strategy from our AD tool. We write FD for one-sided finite differences, SL for statement level and CL for code list. For TAMC, the postfix ftl denotes the forward mode and ad denotes the reverse mode. 
Table 2. Roe flux CPU timings (in $\mu$ seconds) of the function, $T(F)$, on different platforms

\begin{tabular}{rrrrr}
\hline SGI & ALP & SUN & NAG & FUJ \\
\hline 0.8 & 0.5 & 0.9 & 1.8 & 6.2 \\
\hline
\end{tabular}

Timings are based on 10,000 evaluations for each method and an average of 10 runs of the executables from the different machines we used. We have checked the accuracy by evaluating the largest difference from the corresponding derivative found by ADIFOR [4]. This was less than $10^{-14}$ in all cases except finite differences, where it was about $10^{-7}$, in line with the truncation error associated with this approximation.

Table 3. Ratios of Jacobian to Function CPU timings, $T(\nabla F) / T(F)$, on various platforms, ratios of floating point operations, $r_{\text {flops }}=\operatorname{FLOPs}(\nabla F) / \operatorname{FLOPs}(F)$, and numbers of lines of code (\#loc)

\begin{tabular}{|c|c|c|c|c|c|c|c|c|}
\hline No. & Method & SGI & ALP & SUN & NAG & FUJ & $r_{\text {flops }}$ & \#loc \\
\hline 1 & & 2.1 & 12.6 & 12.6 & 13.3 & 11.9 & 11.4 & 176 \\
\hline 2 & VE Forward(SL) & 7.5 & 5.5 & 13.2 & 12.2 & 10.2 & 8.4 & 1534 \\
\hline 3 & VE Rever: & 6 & 4.6 & 8. & 8 & 9 & .9 & 1318 \\
\hline 4 & VE Mark( & 6.4 & 5.1 & 9.8 & 10.4 & 6.7 & 7.2 & 1267 \\
\hline 5 & VE Mark & 6.6 & 5.0 & 14.1 & 10.2 & 6.6 & 7.2 & 1261 \\
\hline 6 & $\mathrm{VE}$ & 6.0 & 4.5 & 8.9 & 9.1 & 6.1 & 6.5 & 1172 \\
\hline 7 & $\mathrm{VE} \mathrm{V}$ & 6.2 & 4.6 & 9.0 & 9.6 & 6.0 & 6.5 & 1170 \\
\hline 8 & VE Forwa & 7.3 & 5.2 & 18.7 & 19.2 & 14.9 & 12.1 & 3175 \\
\hline 9 & VE $\mathrm{F}$ & 6.7 & 4.7 & 10.7 & 9.9 & 9.5 & 8.9 & 2433 \\
\hline 10 & VE Mark( & 6.6 & 5.0 & 9.3 & 11.7 & 11.2 & 7.9 & 2058 \\
\hline 11 & VE Mark & 6.6 & 5.3 & 10.2 & 11.0 & 10.9 & 7.7 & 2012 \\
\hline 12 & VE VL & 6.4 & 5.0 & 10.1 & 11.1 & 10.9 & 7.8 & 2116 \\
\hline 13 & VE VLR RB(CL) & 7.2 & 5.4 & 10.8 & 12.4 & 12.1 & 8.4 & 2145 \\
\hline 14 & ADIF & 5.9 & 9.8 & 31.4 & 50.5 & 14.4 & 15.0 & 614 \\
\hline 15 & TAM & 14.4 & 10.2 & 11.9 & 56.7 & 14.9 & 19.9 & 639 \\
\hline 16 & TAMC-ad & 12.2 & 8.5 & 13.2 & 42.0 & 10.0 & 11.9 & 919 \\
\hline
\end{tabular}

Table 3 shows that on the SGI and ALP platforms the vertex elimination approach for computing Jacobians is about twice as fast as both conventional AD tools and finite differences. On the SUN, NAG and FUJ platforms the vertex elimination approach does not reach the same level of efficiency. We observe that derivative codes for the code list version are often slightly slower than their counterparts differentiated at statement level for ALP and SGI and sometimes much slower on SUN, NAG and FUJ. Denoting by $\operatorname{FLOPs}(F)$ and FLOPs $(\nabla F)$ the numbers of floating point operations required to compute respectively the 
function and its Jacobian, we obtained the ratios also shown in Table 3 from the Roe flux test case. We see that on the SGI and ALP platforms the vertex elimination derivative code ran faster than the ratio of floating point operations predicts. On the SUN, NAG and FUJ platforms the reverse is usually true.

\section{Performance Issues}

The elimination strategies we used are based on criteria aimed at minimising the number of floating point multiplications required to accumulate the Jacobian. Van der Wijngaar and Saphir [15] have shown that, on RISC platforms, neither the number of floating point operations nor even the cache miss rate are sufficient to explain performance of a numerical code; and processor-specific instructions (which can be seen from the generated assembly code) and compiler maturity affect performance.

\subsection{Floating Point Performance}

If we consider the derivative code speed in terms of floating point operations per clock cycle as shown in Fig. 2, all the elimination strategies are performing over 1 floating point operation per cycle on the SGI and ALP platforms. The Compaq EV6 and R12000 can perform up to 2 floating point operations per clock cycle [16] and a throughput of in excess of one floating point operation per clock cycle may be considered highly satisfactory. On the SUN and NAG platforms a throughput of less than half a floating point operation per clock cycle is achieved. Again the theoretical maximum is 2. It was initially thought that cache misses may explain the poor timing ratios on these platforms.

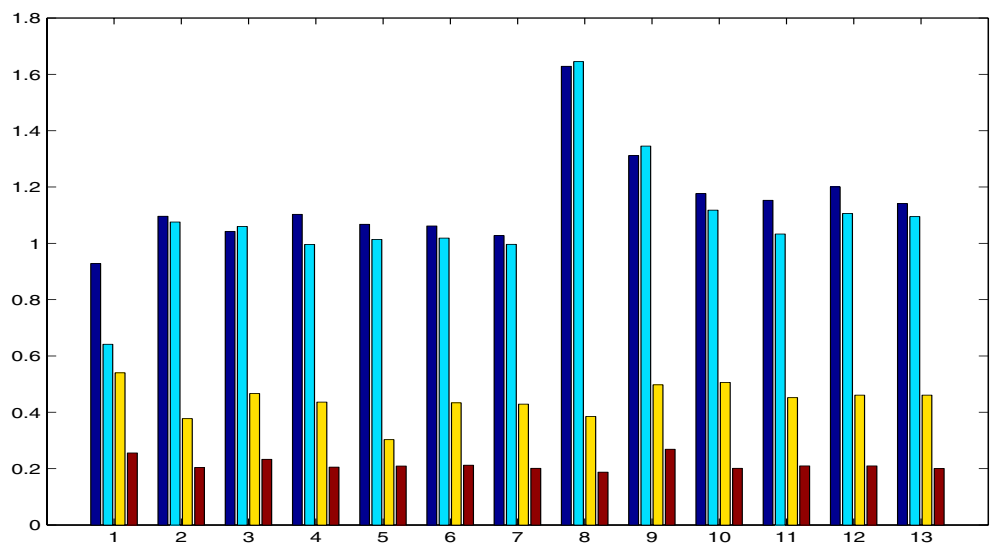

Fig. 2. Number of floating point operations per cycle performed by FD (number 1) and the elimination strategies (numbered 2-13 as they appear in Table 3); the bars represent (from left to right) results obtained from SGI, ALP, SUN, and NAG 


\subsection{Cache Misses}

On the SGI, the outputs from SGI Origin's SpeedShop profiler showed that the derivative codes fit in the instruction cache and they differed from each other only by few cache misses in the primary/secondary data caches. The Compaq Alpha EV6 processor we used has caches comparable to those of the MIPS R12000 (see Table 1). Therefore, we do not expect cache misses to be crucial for the SGI and ALP platforms.

The Sparc Ultra 10 has relatively small caches compared with the R12000 and EV6. On the SUN and NAG platforms we noticed a performance degradation mainly of the code list method. Here, the forward elimination from the code list showed particularly poor performance. Profiling with the Sun Workshop 6.2 tools, we observed a $28 \%$ instruction cache miss rate for this forward elimination strategy. Using a SUN Blade $1000(600 \mathrm{MHz})$ with double the primary cache size, that percentage came down to $10 \%$. Interestingly the timing ratios remained similar to those from the Ultra 10 indicating that although instruction cache misses were occurring, the root cause of the poor performance lay elsewhere.

\subsection{Statement Ordering in the Derivative Code}

The SGI and ALP processors may perform 2 floating point operations per clock cycle through their floating point pipeline, provided that the floating point operation being performed uses data currently in a register and does not require any data from the output of an operation ahead of it and still in the pipeline. The SGI platform has a latency of 2 clock cycles before the result of an operation is available whereas for the ALP platform this is 4 clock cycles [16]. This may explain the slightly better results from the SGI compared to the ALP in Fig. 2.

Apart from their larger cache sizes the other main difference between the SGI/ALP platforms and the SUN/NAG/FUJ platforms is that the SGI and ALP processors support out-of-order execution [16]. This technique involves maintaining queues of floating point operations ready to be performed and if the one at the head of the current queue requires a value currently being processed in the floating point pipeline or not currently in a register, then the processor switches to another queue. Use of this technique reduces the importance of instruction scheduling by the compiler.

On the Ultra10 and Ultra1 processors of the SUN, NAG and FUJ platforms there is no out-of-order execution. The optimising compiler must perform more intensive optimisation of instruction scheduling in order to maintain good use of the floating point pipeline. The derivative codes we produce are large (see final column of Table 3), contain no loops or branching and hence comprise one large basic block. We therefore expected optimising compilers to schedule floating point instructions effectively since there are no complications regarding the control flow of the program. Since this is obviously not occurring for the SUN, NAG and FUJ platforms, we conjectured that the local optimisation of instruction scheduling performed by the compiler might not be able to maintain a good throughput in the floating point pipeline since statements using the 
same variables might be separated by several hundred lines of source code. Since an elimination sequence produces a certain set of elimination statements that may be placed in any order that respects their dependencies, we additionally conjectured that we might be able to reorder the statements in the derivative code to make better use of variables currently in registers.

To assess the impact of statement ordering in the derivative code, we perturbed the order of statements without altering the data dependencies within the code. We reordered the assignment statements with the aim of using each assigned value soon after its assignment. We therefore used a modified version of the depth first search algorithm [17]. Namely, we regarded the statements in the derivative code as the vertices of an acyclic graph, with the output statements at the top and an edge from $s$ to $t$ if statement $t$ uses the output from statement $s$. Then, we arranged the statements in the order produced by a depth-first traversal of this graph. The results are shown in Table 4, which also displays the Table 3 results in brackets. We can see that statement reordering has greatly improved many of the SUN, NAG and FUJ times, has improved most of the SGI times, but has made no significant difference to the ALP times. For instance, looking at the SUN column of Table 4, the statement reordering has improved the generated code using the forward elimination from the code list by $56 \%$. In this case, we observed that the resulting reordered derivative has $34 \%$ less loads and $72 \%$ less stores than the original derivative code. This indicates that the compiler was not achieving efficient register usage for the original derivative code. We now see that the Jacobian to function CPU time ratios have all significantly improved and we are approaching twice the efficiency of the best of the conventional techniques, AD or finite-differencing, of Table 3.

Table 4. Ratios, $T(\nabla F) / T(F)$, of the reordered derivative codes from the Roe flux test case on different platforms, timings in brackets are those before the reordering (c.f. Table 3)

\begin{tabular}{lrllllllllrr}
\hline Method & \multicolumn{2}{c}{ SGI } & \multicolumn{2}{c}{ ALP } & \multicolumn{2}{c}{ SUN } & \multicolumn{2}{c}{ NAG } & \multicolumn{2}{c}{ FUJ } \\
\hline VE Forward(SL) & 6.7 & $(7.5)$ & 5.4 & $(5.5)$ & 9.3 & $(13.2)$ & 7.8 & $(12.2)$ & 7.4 & $(10.2)$ \\
VE Reverse(SL) & 5.2 & $(6.5)$ & 4.6 & $(4.6)$ & 7.7 & $(8.8)$ & 7.2 & $(8.8)$ & 6.2 & $(6.9)$ \\
VE Mark(SL) & 5.8 & $(6.4)$ & 5.2 & $(5.1)$ & 8.6 & $(9.8)$ & 7.3 & $(10.4)$ & 6.1 & $(6.7)$ \\
VE Mark RB(SL) & 5.8 & $(6.6)$ & 5.1 & $(5.0)$ & 7.9 & $(14.1)$ & 6.8 & $(10.2)$ & 6.0 & $(6.6)$ \\
VE VLR(SL) & 6.4 & $(6.0)$ & 4.6 & $(4.5)$ & 8.2 & $(8.9)$ & 6.3 & $(9.1)$ & 5.6 & $(6.1)$ \\
VE VLR RB(SL) & 6.8 & $(6.2)$ & 4.5 & $(4.6)$ & 7.5 & $(9.0)$ & 6.2 & $(9.6)$ & 5.6 & $(6.0)$ \\
VE Forward(CL) & 6.2 & $(7.3)$ & 5.2 & $(5.2)$ & 8.2 & $(18.7)$ & 13.3 & $(19.2)$ & 8.5 & $(14.9)$ \\
VE Reverse(CL) & 6.4 & $(6.7)$ & 5.0 & $(4.7)$ & 8.8 & $(10.7)$ & 7.5 & $(9.9)$ & 7.4 & $(9.5)$ \\
VE Mark(CL) & 6.6 & $(6.6)$ & 5.1 & $(5.0)$ & 7.8 & $(9.3)$ & 7.3 & $(11.7)$ & 6.1 & $(11.2)$ \\
VE Mark RB(CL) & 6.3 & $(6.6)$ & 5.4 & $(5.3)$ & 8.6 & $(10.2)$ & 7.5 & $(11.0)$ & 6.1 & $(10.9)$ \\
VE VLR(CL) & 7.1 & $(6.4)$ & 4.9 & $(5.0)$ & 7.5 & $(10.1)$ & 6.8 & $(11.1)$ & 6.2 & $(10.9)$ \\
VE VLR RB(CL) & 6.8 & $(7.2)$ & 5.8 & $(5.4)$ & 8.1 & $(10.8)$ & 8.1 & $(12.4)$ & 6.4 & $(12.1)$ \\
\hline
\end{tabular}




\section{Conclusions}

Automatic differentiation via vertex elimination allows us to produce Jacobian code which, in conjunction with an optimising compiler, efficiently uses a floating point pipeline on processors that support out-of-order execution. On other platforms tested and that supported a floating point pipeline we found that the compiler was not able to schedule the floating point operations with enough register re-use to allow the floating point processor to perform efficiently. Application of a simple statement reordering strategy based on depth first traversal enabled the compiler to optimise the resulting derivative code more effectively.

We conclude that we have an AD tool that, when assisted by statement reordering on some platforms, produces Jacobian code approaching or exceeding twice the efficiency of the current state-of-the-art for the Roe flux test problem. We are currently testing and evaluating the tool's performance on a number of test problems taken from the MINPACK-2 optimisation test suite [18].

\section{Acknowledgments}

We thank EPSRC and UK MOD for funding this project under grant GR/R21882.

\section{References}

1. Griewank, A., Corliss, G.: Automatic Differentiation of Algorithms. SIAM, Philadelphia (1991)

2. Griewank, A.: Evaluating Derivatives: Principles and Techniques of Algorithmic Differentiation. SIAM, Philadelphia (2000)

3. Corliss, G., Faure, C., Griewank, A., Hascoët, L., Naumann, U., eds.: Automatic Differentiation of Algorithms: From Simulation to Optimization. Springer (2002) (to appear).

4. Bischof, C., Carle, A., Khademi, P., Mauer, A.: ADIFOR 2.0: Automatic differentiation of Fortran 77 programs. IEEE Computational Science \& Engineering 3 (1996) 18-32

5. Faure, C., Papegay, Y.: Odyssée user's guide, version 1.7. Technical Report 0224, INRIA, Unité de Recherche, INRIA, Sophia Antipolis, 2004 Route des Lucioles, B.P. 93, 06902, Sophia Antipolis Cedex, France (1998) See http://www.inria.fr/safir/SAM/Odyssee/odyssee.html.

6. Giering, R., Kaminski, T.: Recipes for adjoint code construction. ACM Transactions on Mathematical Software 24 (1998) 437-474

7. Tadjouddine, M., Forth, S.A., Pryce, J.D., Reid, J.K.: On the Implementation of AD using Elimination Methods via Source Transformation: Derivative code generation. AMOR Report 01/4, Cranfield University (RMCS Shrivenham), Swindon SN6 8LA, England (2001)

8. Tadjouddine, M., Pryce, J.D., Forth, S.A.: On the Implementation of AD using Elimination Methods via Source Transformation. AMOR Report 00/8, Cranfield University (RMCS Shrivenham), Swindon SN6 8LA, England (2000)

9. Parr, T., Lilly, J., Wells, P., Klaren, R., Illouz, M., Mitchell, J., Stanchfield, S., Coker, J., Zukowski, M., Flack, C.: ANTLR Reference Manual. Technical report, MageLang Institute's jGuru.com (2000) See http://www.antlr.org/doc/. 
10. Griewank, A., Reese, S.: On the calculation of Jacobian matrices by the Markowitz rule. [1] 126-135

11. Naumann, U.: Efficient Calculation of Jacobian Matrices by Optimized Application of the Chain Rule to Computational Graphs. PhD thesis, Technical University of Dresden (1999)

12. Naumann, U.: Elimination techniques for cheap Jacobians. [3] 241-246

13. Roe, P.L.: Approximate Riemann solvers, parameter vectors, and difference schemes. Journal of Computational Physics 43 (1981) 357-372

14. Tadjouddine, M., Forth, S.A., Pryce, J.D.: AD tools and prospects for optimal AD in CFD flux Jacobian calculations. [3] 247-252

15. der Wijngaart, R.F.V., Saphir, W.C.: On the efficacy of source code optimizations for cache-based processors. NAS Technical Report NAS-00-014, NASA (2000)

16. Goedecker, S., Hoisie, A.: Performance Optimization of Numerically Intensive Codes. SIAM Philadelphia (2001)

17. Knuth, D.E.: The Art of Computer Programming, Volume 1: Fundamental Algorithms. Adison-Wesley (1997)

18. Averick, B.M., Moré, J.J.: User guide for the MINPACK-2 test problem collection. Technical Memorandum ANL/MCS-TM-157, Argonne National Laboratory, Argonne, Ill. (1991) Also issued as Preprint 91-101 of the Army High Performance Computing Research Center at the University of Minnesota. 